\title{
Exact Travelling Wave Solutions of Nonlinear Wave equations Using Tanh-function Method
}

\author{
Weishi Yin ${ }^{1, a}$, Yixian Gao ${ }^{2, b \text {, * }}$ \\ ${ }^{1}$ School of Science, Changchun university of Science and Technology, Jilin 130022, China \\ ${ }^{2}$ School of Mathematics and Statistics, Northeast Normal University, Jilin 130024, China \\ ayinweishi@foxmail.com, bgaoyx@nenu.edu.cn
}

Keywords: Traveling wave solutions; Tanh-function method; Wave equation.

\begin{abstract}
This paper is concerned with the exact traveling wave solutions of nonlinear wave equations. Using the tanh function method, we can obtain the accurate expression of the solutions. Further, according to the images of the solutions, we can get the variation depends on the velocity $v$.
\end{abstract}

\section{Introduction}

It is well known that nonlinear phenomena are very important in a variety of scientific fields, especially in fluid mechanics, solid state physics, plasma physics, plasma waves, capillary-gravity waves, and chemical physics. Most of these phenomena are described by nonlinear partial differential equations. Analytical solutions of this problems are usually not available, especially when the nonlinear terms are involved. Therefore, finding its travelling solutions is of practical importance.

The methods of looking for exact traveling wave solutions of nonlinear evolution equations, has been tremendous development in recent decades, such as inverse scattering method [2], HI Rota's bilinear technique [5], the Painlve expansion method [13]. In the early nineties of last century, Huibin and Kelin [7] proposed a new method. The main idea of this method is taking hyperbolic tangent function of the power series as possible traveling wave solutions of the nonlinear evolution equations. Then they substituted the power series directly to KdV equation, and obtained the coefficients of the power series. However this method involved very complicated algebra computation. In order to reduce the complex algebra computation, Malfiety [9-11] proposed the tanh-function method. Since all the derivatives of hyperbolic tangent can be expressed by the hyperbolic tangent in itself, this simple translation makes the method can be applied to more nonlinear evolution equations. Fan et al. [3] proposed the extended hyperbolic tangent method, which replace the tanh-function by the solutions of Riccati equation. In [1, 4, 14, 16, and 17], using the tanh function method, they got the exact form of traveling wave solutions of various types of evolution equations. In recent years, the $G^{\prime}$ /G function method [15], the auxiliary function method [6] is based on tanh-function method. This shows that the hyperbolic tangent function method is very effective and direct method when looking for the exact traveling wave solutions of nonlinear evolution equations.

\section{The Tanh-function Method}

Let's consider the nonlinear partial differential equations

$$
N\left(u, u_{t}, u_{x}, u_{x x}, u_{x x x}, \cdots \cdots\right)=0
$$

Where $u(x, t)$ is the real function on $R^{2}$ ? At first, we assume the traveling wave solutions of (2.1) are the form of

$$
u(\mathrm{x}, \mathrm{t})=\mathrm{U}(\omega)=\mathrm{U}(\mathrm{c}(\mathrm{x}-v t)) \text {, }
$$

With the velocity $v$, and the constant c. Submitted (2.2) into (2.1), we can get the ODEs

About $\omega$

$N\left(\mathrm{U}, \mathrm{U}^{\prime}, \mathrm{U}^{\prime \prime}, \mathrm{U}^{\prime \prime \prime}, \ldots\right)=0$.

Second, we assume the possibly traveling wave solutions can be written 
$u(\mathrm{x}, \mathrm{t})=\mathrm{U}(\omega)=\mathrm{H}(\mathrm{Y})=\sum_{i=0}^{K} a_{i} Y^{i}$,

Where $Y=\tanh (\omega)=\frac{e^{\omega}-e^{-\omega}}{e^{\omega}+e^{-\omega}}$ the highest order K will be determined late. Then we can;

Get;

$$
\begin{aligned}
& \frac{d Y}{d \omega}=1-Y^{2}, \frac{d U}{d \omega}=\left(1-\mathrm{Y}^{2}\right) \mathrm{H}^{\prime}, \frac{d^{2} U}{d \omega^{2}}=\left(1-\mathrm{Y}^{2}\right)\left(-2 Y . H^{\prime}+\left(1-\mathrm{Y}^{2}\right) \mathrm{H}^{\prime \prime}\right), \\
& \frac{d^{3} U}{d \omega^{3}}=\left(1-\mathrm{Y}^{2}\right)\left(\left(6 Y^{2}-2\right) H^{\prime}+6 Y\left(1-\mathrm{Y}^{2}\right) \mathrm{H}^{\prime \prime}+\left(1-Y^{2}\right)^{2} H^{\prime \prime \prime}\right),
\end{aligned}
$$

Submitted above equations into (2.3), we can get the ODEs with $\mathrm{Y}$

$N\left(\mathrm{Y}, \mathrm{H}, \mathrm{H}^{\prime}, \mathrm{H}^{\prime \prime}, \mathrm{H}^{\prime \prime \prime}, \ldots\right)=0$

Where $H^{\prime}=\frac{d H}{d Y}$ To determine the parameter $\mathrm{K}$, we usually balance the nonlinear term and the highest order derivative term in equation (2.5). Then, we submitted (2.4) (with the determined K) into (2.5), and get the polynomial equation with Y. Collecting all the coefficients of power of Y, and letting the coefficients of each power of $\mathrm{Y}$ to be vanished, we can determined all the coefficient $a_{1}, a_{2}, \cdots \cdots, a_{K}$. According to (2.4), we can get the traveling wave solutions of (2.1).

\section{The Nonlinear Wave Equations}

Consider the nonlinear wave equations;

$u_{t t}=u_{x x}+u\left(1-u^{2}\right)$.

Submitted (2.2) into (3.1), we can get;

$c^{2} v^{2} U^{\prime \prime}=c^{2} U^{\prime \prime}+U\left(1-\mathrm{U}^{2}\right)$.

According to (2.5), we have;

$c^{2} v^{2} U^{\prime \prime}\left(-2 \mathrm{YH}^{\prime}+\left(1-\mathrm{Y}^{2}\right) \mathrm{H}^{\prime \prime}\right)=c^{2}\left(1-\mathrm{Y}^{2}\right)\left(-2 Y H^{\prime}+\left(1-Y^{2}\right) \mathrm{H}^{\prime \prime}\right)+\mathrm{H}\left(1-\mathrm{H}^{2}\right)$.

Balancing $Y^{4} H^{\prime \prime}$ ' with $H^{3}$, yields $4+K-2=3 K$ i.e. $\mathrm{K}=1$. Thus $H(Y)=a_{0}+a_{1} Y \quad H^{\prime}=a_{1}, H^{\prime \prime}=0$.

Submitting it into above equation, we have

$-2 a_{1} c^{2} v^{2} Y+2 a_{1} c^{2} v^{2} Y^{3}=-2 a_{1} c^{2} Y+2 a_{1} c^{2} Y^{2}+\left(a_{0}+a_{1} \mathrm{Y}\right)\left(1-\left(a_{0}+a_{1} \mathrm{Y}\right)^{2}\right)$.

Collecting the coefficients of each power of $\mathrm{Y}$, we get the algebraic for $a_{0}, a_{1}, c, v$ :

$$
\left\{\begin{array}{c}
a_{0}-a_{0}^{3}=0 \\
2 a_{1} c^{2} v^{2}-2 a_{1} c^{2}-3 a_{0}^{2} a_{1}+a_{1}=0 \\
-3 a_{0} a_{1}^{2}=0 \\
-2 a_{1} c^{2} v^{2}+2 a_{1} c^{2}-a_{1}^{3}=0
\end{array}\right.
$$

Then, the solutions of (3.2) are

$a_{1}=-1, a_{0}=0, v=-\frac{\sqrt{-1+2 c^{2}}}{\sqrt{2} c} ; a_{1}=-1, a_{0}=0, v=\frac{\sqrt{-1+2 c^{2}}}{\sqrt{2} c} ;$
$a_{1}=1, a_{0}=0, v=-\frac{\sqrt{-1+2 c^{2}}}{\sqrt{2} c} ; a_{1}=-1, a_{0}=0, v=\frac{\sqrt{-1+2 c^{2}}}{\sqrt{2} c}$,

With $c \neq 0$. Recall that $u(\mathrm{t}, \mathrm{x})=\mathrm{U}(\omega)=\mathrm{U}(\mathrm{c}(\mathrm{x}-v t))=a_{0}+a_{1} Y(\omega)=a_{0}+a_{1} \tanh (\mathrm{c}(\mathrm{x}-\mathrm{vt}))$,

Hence we obtain the traveling wave solutions of (3.1)

$u_{1}(\mathrm{x}, \mathrm{t})=-\tanh \left[\mathrm{c}\left(\mathrm{x}+\frac{\sqrt{-1+2 c^{2}}}{\sqrt{2} c} \mathrm{t}\right)\right] ; \mathrm{u}_{2}(\mathrm{x}, \mathrm{t})=-\tanh \left[\mathrm{c}\left(\mathrm{x}-\frac{\sqrt{-1+2 c^{2}}}{\sqrt{2} c} \mathrm{t}\right)\right]$ 
$u_{3}(\mathrm{x}, \mathrm{t})=\tanh \left[\mathrm{c}\left(\mathrm{x}+\frac{\sqrt{-1+2 c^{2}}}{\sqrt{2} c} \mathrm{t}\right)\right] ; \mathrm{u}_{4}(\mathrm{x}, \mathrm{t})=\tanh \left[\mathrm{c}\left(\mathrm{x}-\frac{\sqrt{-1+2 c^{2}}}{\sqrt{2} c} \mathrm{t}\right)\right]$

Since the velocity $v=-\frac{\sqrt{-1+2 c^{2}}}{\sqrt{2} c}$, the constant c must satisfies $c \geq \sqrt{\frac{1}{2}}$. We plot the Images of (3) with specially values $c=\sqrt{\frac{1.000001}{2}}, c=1.5, c=100000$, which the correspond Velocity $\mathrm{v}$ is close to 0 , constant and 1.

$$
\begin{aligned}
& u(\mathrm{x}, \mathrm{t})=-\operatorname{coth}\left[\mathrm{c}\left(\mathrm{x}+\frac{\sqrt{-1+2 c^{2}}}{\sqrt{2} c} \mathrm{t}\right)\right] ; u(\mathrm{x}, \mathrm{t})=-\operatorname{coth}\left[\mathrm{c}\left(\mathrm{x}-\frac{\sqrt{-1+2 c^{2}}}{\sqrt{2} c} \mathrm{t}\right)\right] \\
& u(\mathrm{x}, \mathrm{t})=\operatorname{coth}\left[\mathrm{c}\left(\mathrm{x}+\frac{\sqrt{-1+2 c^{2}}}{\sqrt{2} c} \mathrm{t}\right)\right] ; u(\mathrm{x}, \mathrm{t})=\operatorname{coth}\left[\mathrm{c}\left(\mathrm{x}-\frac{\sqrt{-1+2 c^{2}}}{\sqrt{2} c} \mathrm{t}\right)\right]
\end{aligned}
$$

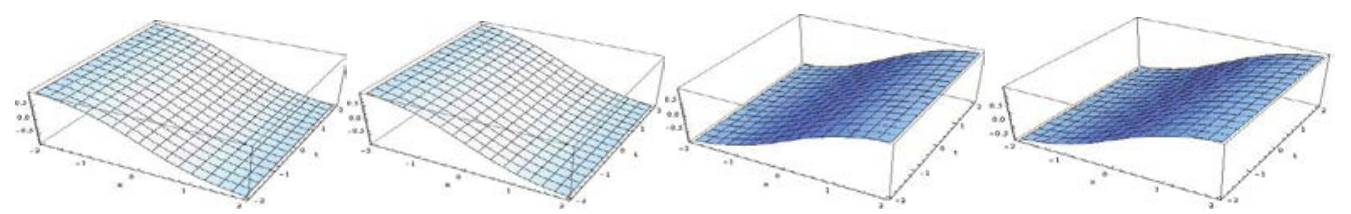

Fig. 1. The travelling wave solutions of (3), when $c=\sqrt{1.000001 / 2},(x, t) \in[-2,2] \times[-2,2]$

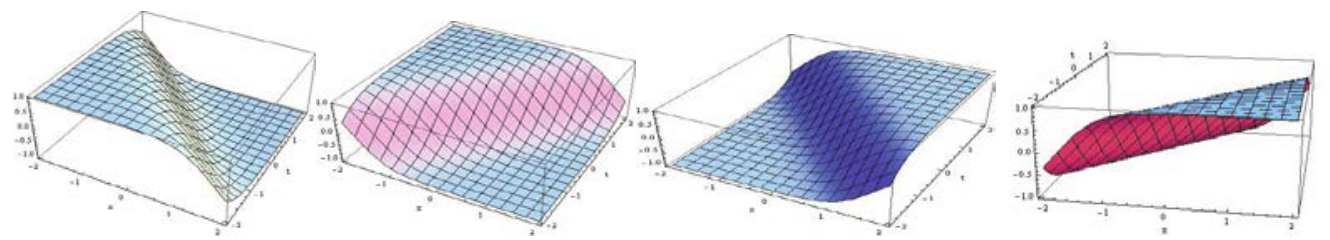

Fig. 2. The travelling wave solutions of (3), when $c=1.5,(\mathrm{x}, \mathrm{t}) \in[-2,2] \times[-2,2]$.

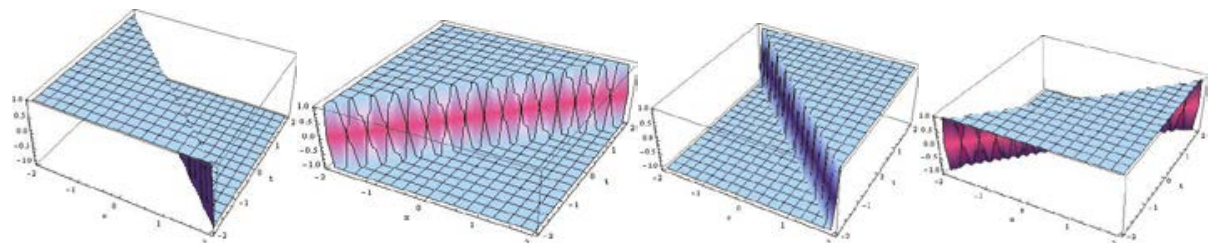

Fig. 3. The travelling wave solutions of (3), when $c=100000,(x, t) \in[-2,2] \times[-2,2]$.

\section{Conclusion}

In this paper, we have applied the tanh-function method to construct a series of traveling wave solutions for nonlinear wave equations. These traveling waves' solutions are expressed in terms of hyperbolic tangent or hyperbolic cotangent functions depending on different parameters. The tanh-function method is direct, concise and effective, which can be applied to many other nonlinear evolution equations.

\section{Acknowledgments}

This work was supported by NSFC Grant (Nos. 11001042, 11171056, 11171130, 11271062, 51278221 and 51378076) and SRFDP Grant (No. 20100043120001.) 


\section{References}

[1] M.A. Abdou, New exact travelling wave solutions using modified extended tanh-function method, Chaos, Solitons and Fractals, 31 (2007), 840-85.

[2] M.J. Ablowitz, P.A. Clarkson, Solitons, Nonlinear Evolution and Inverse Scattering, Cambridge Univ. Press, 1991.

[3] E. Fan, Y. Hon, Generalized tanh method extended to special types of nonlinear equations, Z. Naturforsch, 57 (2002) 692-700.

[4] K. Glasner, Nonlinear preconditioning for diffuse interfaces, J. Compute. Phys. 174 (2001), 695-711.

[5] C.H. Gu, Soliton Theory and Its Application, Springer, Berlin, 1995.

[6] D. Huang, D. Li, H. Zhang, Explicit and exact travelling wave solutions for the generalized derivative Schrodinger equation, Chaos, Solitons and Fractals, 31 (2007), 586-59.

[7] L. Huibin, W. Kelin, Exact solutions for two nonlinear equations: I, J. Phys. A: Math. Gen., 23(1990), 3923-3928.

[8] A.H. Khater, W. Malfliet, D. K. Callebaut, E. S. Kamel, The tanh method, a simple transformation and exact analytical solutions for nonlinear reaction-diffusion equations, Chaos Soliton. Fact, 14 (2002), 513-522.

[9] W. Malfliet, Solitary wave solutions of nonlinear wave equations, Am. J. Phys. 60 (7), (1992), 650-654.

[10] W. Malfliet, The tanh method: I. Exact solutions of nonlinear evolution and wave equations, Physica Scripta, 54 (1996), 563-568.

[11] W. Malfliet, The tanh method: II. Perturbation technique for conservative systems, Physica Scripta, 54 (1996), 569-575.

[12] E.J. Parkes, B.R. Duffy, An automated tanh-function method for finding solitary wave solutions to non-linear evolution equations, compute. Phys. Commun, 98 (1996), 288-300.

[13] B.Tian, Y. Gao, Truncated Pain eve expansion and a wide-ranging type of generalized variable-coefficient Kadomtsev-Petviashvili equations, Phys. Let. A, 209 (1995), 297-304.

[14] M. Wang, Exact solutions for a compound KdV-Burgers equation, Phys. Lett. A, 213 (1998), 279-287.

[15] M. Wang, X. Li, J. Zhang, the (G '/G)-expansion method and travelling wave solutions of nonlinear evolution equations in mathematical physics, Phys. Lett. A, 372 (2008), 417-42.

[16] A.M. Wazwaz, Partial Differential Equations: Methods and Applications, Balkema, the Netherlands, 2002.

[17] A.M. Wazwaz, The tanh method for traveling wave solutions of nonlinear equations, applied Mathematics and Computation, 154 (2004), 713-72. 